\title{
Functional polymorphisms of the APOA1/C3/A4/A5-ZPR1- BUD13 gene cluster are associated with dyslipidemia in a sex- specific pattern
}

\author{
Wei Bai ${ }^{1}$, Changgui Kou ${ }^{1}$, Lili Zhang ${ }^{1}$, Yueyue You ${ }^{1}$, Weiying $\mathbf{Y u}^{1}{ }^{1}$, Wanqing Hua ${ }^{1}$, Yuanyuan $\operatorname{Li}^{1}$, Yaqin \\ Yu ${ }^{1}$, Tiancheng Zhao ${ }^{\text {Corresp., }}{ }^{2}$, Yanhua Wu ${ }^{\text {Corresp. } 1,3}$ \\ 1 Department of Epidemiology and Biostatistics, School of Public Health, Jilin University, Changchun, Jilin Province, China \\ 3 Division of Clinical Research, First Hospital of Jilin University, Changchun, Jilin Province, China \\ Corresponding Authors: Tiancheng Zhao, Yanhua Wu \\ Email address: zhaotiancheng@jlu.edu.cn, wuyanhua@jlu.edu.cn
}

Background. Dyslipidemia contributes to the risk of many diseases, including stroke, cardiovascular disease and metabolic-related diseases. Previous studies have indicated that single nucleotide polymorphisms (SNPs) are associated with different levels of serum lipid. Therefore, this study explored the relationship between the APOA1/C3/A4/A5-ZPR1-BUD13 gene cluster gene polymorphisms and dyslipidemia in the total sample population and stratified by genders in a northeast Chinese population.

Methods. A total of 3850 participants from Jilin Province, China, were enrolled in our study, and their serum lipid levels were measured. Six functional SNPs (APOA1 rs5072, APOC3 rs5128, APOA4 rs5104, APOA5 rs651821, ZPR1 rs2075294 and BUD13 rs10488698) were genotyped using polymerase chain reaction and MALDI-TOF-MS. Logistic regression analysis was performed to explore the relationship of APOA1/C3/A4/A5-ZPR1-BUD13 gene cluster gene polymorphisms with dyslipidemia. Linkage disequilibrium and haplotype analyses were performed with SNPStats program and Haploview software.

Results. All SNPs conformed to Hardy-Weinberg Equilibrium. Logistic regression analysis revealed that rs5072, rs5128 and rs651821 were associated with hypertriglyceridemia, rs5104 and rs651821 were associated with low-HDL cholesterolemia in overall group. rs651821 was associated with hypertriglyceridemia and low-HDL cholesterolemia in both the male and female group. However, among females, rs5072 was observed to be associated with hypertriglyceridemia. Haplotype analysis showed that haplotypes TGCCGC and CAGCGC were associated with dyslipidemia in the overall, male and female groups.

Conclusion. SNPs in the APOA1/C3/A4/A5-ZPR1-BUD13 gene cluster were associated with dyslipidemia. Furthermore, the association of $A P O A 1$ rs5072 in this gene cluster with dyslipidemia differed between genders; thus, additional studies are needed to confirm this conclusion, and the mechanisms underlying these results warrant further exploration. 
1 Functional polymorphisms of the APOA1/C3/A4/A5-ZPR1-BUD13 gene cluster are 2 associated with dyslipidemia in a sex-specific pattern

3 Wei Bai ${ }^{1}$, Changgui Kou ${ }^{1}$, Lili Zhang ${ }^{1}$, Yueyue You ${ }^{1}$, Weiying $\mathrm{Yu}^{1}$, Wanqing Hua ${ }^{1}$, Yuanyuan

$4 \mathrm{Li}^{1}$, Yaqin $\mathrm{Yu}^{1}$, Tiancheng $\mathrm{Zhao}^{2 *}$, Yanhua $\mathrm{Wu}^{1,3^{*}}$

5 1. Department of Epidemiology and Biostatistics, School of Public Health, Jilin University, 1163

6 Xinmin Street, Changchun, 130021, Jilin province, China

7 2. Department of Endoscopy Center, China-Japan Union Hospital of Jilin University, 8 Changchun, 130033, Jilin province, China.

9 3. Division of Clinical Research, First Hospital of Jilin University, Changchun, 130021, Jilin 10 province, China.

11 *Corresponding Authors: Tiancheng Zhao, Yanhua Wu

12 Email address: Tiancheng Zhao, zhaotiancheng@jlu.edu.cn 
55

mechanisms underlying these results warrant further exploration.

Keywords: $A P O A 1 / C 3 / A 4 / A 5-Z P R 1-B U D 13$ gene cluster, single nucleotide polymorphism (SNP), dyslipidemia

\section{Background}

Dyslipidemia, which is characterized by abnormally increased total cholesterol (TC), triglycerides (TG), low-density lipoprotein cholesterol (LDL-C) and decreased high-density lipoprotein cholesterol (HDL-C) levels, is a well-established risk factor for cardiovascular diseases (CVD)(El Hajj et al. 2016; Parhofer 2015; Rai et al. 2016) and contributes to metabolic syndrome (MetS), stroke and other metabolism-related diseases(Albai et al. 2017; Amalakanti et al. 2016; Wang et al. 2017; Wu et al. 2016). A nationally representative survey in China indicated that the prevalence of high TC, high LDL-C, low HDL-C and high TG in Chinese adults over the age of 18 was $6.9 \%, 8.1 \%, 20.4 \%$ and $13.8 \%$, respectively(Zhang et al. 2018). Previous studies involving epidemiological surveys found that diet, exercise and other environmental risk factors may be associated with abnormal serum lipid levels(Tan et al. 2016). However, genetic factors could also partially explain the development of dyslipidemia(Dron \& Hegele 2016; Dron \& Hegele 2018; Weiss et al. 2006).

The APOA1/C3/A4/A5-ZPR1-BUD13 (APO, apolipoprotein; ZPR1, zinc finger; BUD13, BUD13 homolog) gene cluster is located on chromosome 11q23.3, and the apolipoprotein gene cluster at this location has been revealed to be more often associated with lipid traits via genomewide association study (GWAS)(Braun et al. 2012; Willer et al. 2013). APOA1 synthesis and lipolysis promote HDL-mediated reverse cholesterol transport by generating cholesterol acceptors(Reichl \& Miller 1989); therefore, APOA1 plays an important part in lipid metabolism, especially that of HDL cholesterol. The major function of APOC3 in lipid metabolism is to inhibit lipoprotein lipase (LPL); thus, the concentrations of plasma APOC3 are positively associated with TG concentrations(Ooi et al. 2008). APOA4 modulates the activation of LPL by APOC2(Goldberg et al. 1990) and participates in reverse cholesterol transport(Stein et al. 1986). The transcription of APOA4 is regulated synchronously with that of APOA1, supporting the relationship between these 
82

apolipoproteins(Malmendier et al. 1991), and APOA4 is associated with TG and HDL levels. APOA5 is a component of HDL and regulates TG levels via hydrolysis of TG-rich lipoproteins and endocytosis of lipoprotein remnants(Forte et al. 2009). BUD13 encodes the BUD13 homolog protein, which is one of the subunits of the splicing factor that affects nuclear pre-mRNA retention(Brooks et al. 2009). ZPRI encodes a regulatory protein involved in cell proliferation and signal transduction(Galcheva-Gargova et al. 1996). In summary, based on the above mechanisms, this gene cluster can influence lipid levels, therapy contributing to the risk of dyslipidemia.

Relevant studies have been conducted to explore the relationship between serum lipid levels and genetic loci in this gene cluster among different ethnic groups(Aung et al. 2014a; Aung et al. 2014b; Hsueh et al. 2017; Suarez-Sanchez et al. 2017; Yin et al. 2011). However, to the best of our knowledge, there have been few studies conducted in northeast Chinese populations that explore the relationship between the APOA1/C3/A4/A5-ZPR1-BUD13 gene cluster and dyslipidemia. Functional SNP may influence gene and protein expression. This study assessed the association of tag and well-studied SNPs (APOA1 rs5072, APOC3 rs5128, APOA4 rs5104, APOA5 rs651821, ZPR1 rs2075294 and BUD13 rs10488698) selected from the Han Chinese data in Haploview (http://hapmap.ncbi.nlm.nih.gov/) and based on previous studies(Wu et al. 2015; Wu et al. 2016) with dyslipidemia in the overall sample and stratified by genders in a large sample from a Han Chinese population.

\section{Materials and Methods}

\section{Study population}

The Project of Present Situation and Change Forecast of Disease Spectrum in Jilin Province, China, was conducted from June 2012 to August 2012 among individuals aged 18 to 79 years in all nine areas of Jilin Province. This project was a cross-sectional and representative survey of Jilin Province, China, which aimed to evaluate the prevalence and risk factors associated with chronic diseases(Wang et al. 2015). The genetic associations results for dyslipidemia-related diseases have been reported in previous studies(Su et al. 2016; You et al. 2018; You et al. 2017). The whole sample size of the survey was 21435 , and the participants in our present study were randomly 
chosen from the whole sample. They were unrelated members of the Han population, and individuals with a family history of dyslipidemia and other metabolic diseases or without complete serum lipid data were excluded; the participants were excluded if they were being treated with any drugs that may affect lipid parameters. In total, 3850 participants (1927 males and 1923 females) were enrolled in the association study between the $A P O A 1 / C 3 / A 4 / A 5-Z P R 1-B U D 13$ gene cluster and dyslipidemia.

Dyslipidemia was assessed according to the Guidelines on Prevention and Treatment of Dyslipidemia in Chinese Adults(Prevention \& Adults 2007): hypertriglyceridemia, TG $\geq 2.26$ $\mathrm{mmol} / \mathrm{L}$; hypercholesterolemia, TC $\geq 6.22 \mathrm{mmol} / \mathrm{L}$; hyper-LDL cholesterolemia, LDL-C $\geq 4.14$ $\mathrm{mmol} / \mathrm{L}$; low-HDL cholesterolemia, HDL-C $<1.04 \mathrm{mmol} / \mathrm{L}$.

For laboratory evaluation, including the assessment of total TC, TG, HDL-C and LDL-C levels, 5-mL blood samples were collected from each individual. The blood samples were transported under refrigeration and then stored at $-20^{\circ} \mathrm{C}$. Our study was approved by the ethics committee of the Jilin University School of Public Health (2012-R-011), and all subjects in this study signed a written informed consent form.

\section{SNP selection}

Tag SNPs were selected from the Han Chinese data in Haploview (http://hapmap.ncbi.nlm.nih.gov/). Other functional or well-studied SNPs were simultaneously selected based on previous studies( $\mathrm{Wu}$ et al. 2015; Wu et al. 2016) that have documented associations between SNPs in the $A P O A 1 / C 3 / A 4 / A 5-Z P R 1-B U D 13$ gene cluster and lipid levels. The minor allele frequency (MAF) criterion for these SNPs was a MAF greater than 0.05 in the Chinese Han population. Finally, 6 SNPs were included in this study: APOA1 rs5072, APOC3 rs5128, APOA4 rs5104, APOA5 rs651821, ZPR1 rs2075294 and BUD13 rs10488698.

\section{DNA extraction and genotyping}

Genomic DNA was extracted from peripheral blood lymphocytes following the protocols provided with a commercial DNA extraction kit (JiuNa biology, Hangzhou, China). Genotyping of each SNP was performed via MALDI-TOF-MS (Sequenom, San, Diego, CA, USA) with a 
136

137

138

139

140

141

142

143

144

145

146

147

148

149

150

151

152

153

154

155

156

157

158

159

160

161

162

MassARRAY system. Completed genotyping reactions were dispensed onto a 384-well SpectroCHIP using a MassARRAY nanodispenser (Sequenom).

The detection rates for the 6 SNPs were $99.7 \%, 99.6 \%, 95.8 \%, 99.6 \%, 99.8 \%$, and $99.8 \%$ (for APOA1 rs5072, APOC3 rs5128, APOA4 rs5104, APOA5 rs651821, ZPR1 rs2075294 and BUD13 rs 10488698, respectively).

\section{Statistical analysis}

Continuous variables with a normal distribution were summarized as the mean \pm standard deviation and compared by Student's t-test. The Chi-square test was used to check whether the genotype and allele distributions of the six SNPs were significantly different between males and females. For each SNP, Hardy-Weinberg Equilibrium (HWE) was calculated with the goodnessof-fit Chi-square test. $P<0.05$ was considered statistically significant. Binary logistic regression analysis was used to detect associations between the genotypes of the six SNPs in the APOA1/C3/A4/A5-ZPR1-BUD13 gene cluster (in Dominant Model) and dyslipidemia adjusted for sex (adjusted only in the overall group), age, BMI and waist circumference in the overall, male and female groups. We set hypercholesterolemia, hypertriglyceridemia, high LDL cholesterolemia and reduced HDL cholesterolemia as dependent variables, while genotypes, sex (only in the overall group), age, BMI and waist circumference were set as independent variables and entered as method. The strength of any evident association was explored by calculating odds ratios together with their $95 \%$ confidence intervals $(\mathrm{CI})$, and $P$ values of no more than 0.008 after Bonferroni Correction were considered statistically significant. All analyses were conducted using SPSS 24.0. Linkage disequilibrium and haplotype analyses were performed with the SNPStats program (http://bioinfo.iconcologia.net/SNPStats)(Sole et al. 2006) and Haploview software(Barrett et al. 2005).

\section{Results}

\section{Subject characteristics and distributions of genotype and allele}

The baseline characteristics of the subjects stratified by genders were shown in Table 1. No difference was observed between males and females in terms of BMI, but the average age and 
163

164

165

166

167

168

169

170

171

172

173

174

175

176

177

178

179

180

181

182

183

184

185

186

187

188

189

waist circumference were greater in males than females. In addition to the percentage of subjects with hypercholesterolemia, there were significant differences in the percentages of subjects with hypertriglyceridemia, high LDL, reduced HDL and dyslipidemia between males and females. There were 2264 participants without dyslipidemia while the numbers of participants with one to four type of dyslipidemia were 763, 623,184 and 16, respectively. The top five types of dyslipidemia (and the number of participants with each type) were as follows: hypertriglyceridemia (425), hypertriglyceridemia + reduced HDL cholesterolemia (397), reduced HDL cholesterolemia (220), hypercholesterolemia + high LDL cholesterolemia (126) and hypertriglyceridemia + hypercholesterolemia + high LDL cholesterolemia (107).

The genotype distributions of the six SNPs in the APOA1/C3/A4/A5-ZPR1-BUD13 gene cluster conformed to HWE in the overall group (Table S1). The allele distribution of one SNP (rs651821) was different between males and females, and the genotype and allele distributions of the six SNPs were shown in Table S1. Genotype distributions and allele frequencies of dyslipidemia and normal groups were shown in Table S2.

\section{Association between SNPs and dyslipidemia}

In the overall group, logistic regression analysis revealed that dyslipidemia was associated with three SNPs (rs5072, rs5104 and rs651821) after adjusting for sex, age, BMI and waist circumference (Table 2). In the male group, rs651821 was found to be associated with hypertriglyceridemia $(\mathrm{OR}=1.78,95 \% \mathrm{CI}=1.41-2.26)$ and low-HDL cholesterolemia $(\mathrm{OR}=1.61$, 95\% $\mathrm{CI}=1.27-2.05)$. Similar results were observed in the female group. However, we found that rs5072 was associated with hypertriglyceridemia $(\mathrm{OR}=1.39,95 \% \mathrm{CI}=1.09-1.76)$ in the female group (Table 2). The association between the numbers of risk alleles and dyslipidemia was shown in Table S3.

\section{Linkage disequilibrium (LD) and haplotype analyses}

The pattern of pairwise linkage disequilibrium (LD) between the SNPs of the APOA1/C3/A4/A5-ZPR1-BUD13 gene cluster was shown in Figure 1. Rs10488698, rs2075294 and rs651821 were located in block 1, while rs5104, rs5128 and rs5072 were located in block 2 
190 after choosing the cut-off value of 0.75 for D'. The haplotype analysis results were shown in Table

3. People carrying haplotypes 2 and 3 presented an increased risk of dyslipidemia in the total sample and the female and male groups. The association between haplotypes in blocks and different types of dyslipidemia stratified by sexes was explored. We found that subjects with haplotype CGC of block1 exhibited a higher risk of developing hypertriglyceridemia and reduced HDL cholesterolemia in both sexes. In males, subjects carrying haplotype GCT of block 2 presented a higher risk of developing hypertriglyceridemia and reduced HDL cholesterolemia; however, these results were not observed in females. Details were shown in Table S4.

\section{Discussion}

The study applied a large sample design to study the association between the $A P O A 1 / C 3 / A 4 / A 5-Z P R 1-B U D 13$ gene cluster gene polymorphisms and dyslipidemia. The participants in this study were enrolled from a representative community-based population. To the best of our knowledge, this is the first study to analyze the association of this gene cluster with dyslipidemia in a sex-specific pattern in northeast China.

The $A P O A 1 / C 3 / A 4 / A 5$ gene cluster is a well-studied gene cluster. Suarez-Sanchez F et al. found that there was a significant association between $A P O A 1$ rs5072 and $\mathrm{TG}$ in Mexican children(Suarez-Sanchez et al. 2017), and in an Indian study(Pranavchand \& Reddy 2017), Pranavchand R concluded that rs5072 was also associated with increased TG; both of these findings were similar to our results. Pranavchand R(Pranavchand \& Reddy 2017) found that the genotypic and allelic frequencies of rs5072 exhibited no significant differences between cases and controls; however, our study indicated that there was an association between them in females. One explanation for the difference between these two studies could be that they were conducted in people of different ethnicities and ages. A recent exome-wide association study (EWAS)(Yamada et al. 2017) performed in a Japanese population indicated that $A P O A 4$ rs5104 located in this gene was associated with the serum TG concentration. A genome-wide association study (GWAS) conducted in Chinese people(Zhou et al. 2013) showed that rs651821 presented a strong association with TG levels. Cha S et al.(Cha et al. 2014) drew the conclusion that subjects carrying 
217 the variant allele of this SNP exhibited a higher risk of developing low-HDL cholesterolemia than 218 noncarriers in both genders in Koreans, which was consistent with our findings. In our study, 219 haplotype analysis showed that participants carrying the CGC haplotype of block 1 presented a 220 higher risk of developing hypertriglyceridemia $(\mathrm{OR}=1.76,95 \% \mathrm{CI}=1.59-1.96)$ and reduced HDL $221(\mathrm{OR}=1.80,95 \% \mathrm{CI}=1.59-2.03)$ than those carrying the CGT haplotype, which revealed that the T

222

223

224

225

226

227

228

229

230

231

232

233

234

235

236

237

238

239

240

241

242

allele of rs651821 was associated with a higher risk of hypertriglyceridemia and reduced HDL. The MAFs of rs5072, rs5128, rs5104 and rs651821 in our study were $0.3060,0.2966,0.2995$, and 0.2406 , respectively. These results were similar to the frequencies identified in the southern Han Chinese population in the 1000 Genomes Project, which were 0.3190, 0.3190, 0.3571 and 0.2714. Compared with the frequencies observed in American subjects $(0.0656,0.0492,0.1311$ and 0.1639), British subjects $(0.1099,0.1209,0.1648$ and 0.0714$)$ and other ethnicities, the frequencies determined in our study were markedly different. Ethnicity may be critically important in studying the association between SNPs and dyslipidemia. We used Polyphen to predict the influence of two missense mutations (APOA4 rs5014 and BUD13 rs10188698) on the resultant proteins and found that there was no significant influence on protein function. We also observed that these two SNPs were not associated with any types of dyslipidemia.

At present, studies on BUD13-ZPR1 gene polymorphisms focus on the association with lipidrelated diseases. $B U D 13$ and $Z P R 1$ were found to be associated across anatomical categories of coronary artery disease in an Indian study(Pranavchand et al. 2017). Another study(Lin et al. 2016) performed in a Taiwanese population indicated that BUD13 may contribute to the risk of metabolic syndrome, and BUD13 rs623908 was shown to be significantly associated with high TG, low HDL, and HDL levels. Among Chinese individuals(Xu et al. 2018), a strong association between BUD13-ZPR1 rs964184 and coronary heart disease was found, and both gender and age had great impacts on the association of the rs964184 polymorphism with coronary heart disease. Another study(Ueyama et al. 2015) indicated that rs964184 was significantly associated with the prevalence of metabolic syndrome. In addition, there have been studies showing that that ZPRI contributed to the risk of type 2 diabetes mellitus(Guan et al. 2016; Tokoro et al. 2015). Due to the association 
244 of these two genes with lipid-related diseases, we conjecture that BUD13 and ZPRI gene 245 polymorphisms may contribute to the risk of dyslipidemia.

246 In our study, we obtained the novel finding of sex-specific differences between the

247

248

249

250

251

252

253

254

255

256

257

258

259

260

261

262

263

264

265

266

267

268

269

270

APOA1/C3/A4/A5-ZPR1-BUD13 gene cluster gene polymorphisms and dyslipidemia. These differences could be partially explained by differences in sex between males and females. Menopause could change the lipid profile by reducing HDL-C and increasing TG, TC and LDLC(Reddy Kilim \& Chandala 2013). However, after menopause, healthy females were prone to become more insulin resistant and gain total body and intra-abdominal fat(Milewicz et al. 2001). Additionally, females with polycystic ovary syndrome (PCOS) usually become overweight or obese, especially abdominally obese(Wu \& von Eckardstein 2003). Accumulation of body and central fat and decreased insulin resistance are associated with TG, HDL-C and LDL-C and may therefore alter lipid homeostasis, respectively(Ferrannini et al. 2007). Consequently, mechanisms underlying sex-specific differences between the gene cluster and dyslipidemia are complex and should be further studied. We also found that the associations of the SNPs with dyslipidemia were different when the total sample, males and females were considered separately. We speculated that these associations may be influenced by sex and $P$ values we set, since some $P$ values were between 0.008 and 0.05 . There are some limitations of our study that must be recognized. In each gene, we selected only one tag SNP with a MAF $>5 \%$ reported in published articles. We may have ignored some SNPs with low MAFs or important functions in the APOA1/C3/A4/A5-ZPRIBUD13 gene cluster. In addition, dyslipidemia is influenced not only by genetic factors but also by diet, exercise and other environmental factors. We adjusted only sex, age, BMI and waist circumference in our study. Consequently, more environmental factors need to be recorded and adjusted in future studies, and the effects and mechanisms of SNPs in relation to gene expression and protein expression warrant further study.

\section{Conclusion}

In conclusion, we analyzed and assessed the association between $A P O A 1 / C 3 / A 4 / A 5-Z P R I-$ BUD13 gene cluster gene polymorphisms and dyslipidemia between sexes. We not only obtained 
271

272

273

274

275

276

277

278

279

280

281

282

283

284

285

286

287

288

289

290

291

292

293

294

295

296

297

298

299

300

301

302

303

304

305

306

307

308

several results consistent with those reported based on previous studies but also found one SNP (rs5072) was different between males and females, providing new insight into the mechanisms of dyslipidemia in different genders.

\section{Acknowledgments}

We are grateful to the Jilin Provincial Health Department and the Chinese Jilin Provincial Center for Disease Control and Prevention for supporting our project.

\section{References}

Albai O, Roman D, and Frandes M. 2017. Hypertriglyceridemia, an important and independent risk factor for acute pancreatitis in patients with type 2 diabetes mellitus. Ther Clin Risk Manag 13:515-522. 10.2147/tcrm.s134560

Amalakanti S, Nagarjunakonda S, Uppala V, Gajula RK, Potharlanka MG, Rajanala L, Athina S, and Daggumati R. 2016. Stroke: Changing Risk Factor Profile and Unaltered Mean Age in a Developing Country. A Retrospective Study and Literature Review. Eur Neurol 75:229235. 10.1159/000445944

Aung LH, Yin RX, Wu DF, Wang W, Wu JZ, and Liu CW. 2014a. Sex-specific association of the zinc finger protein 259 rs2075290 polymorphism and serum lipid levels. Int J Med Sci 11:471-478. 10.7150/ijms.8489

Aung LH, Yin RX, Wu JZ, Wu DF, Wang W, and Li H. 2014b. Association between the MLX interacting protein-like, BUD13 homolog and zinc finger protein 259 gene polymorphisms and serum lipid levels. Sci Rep 4:5565. 10.1038/srep05565

Barrett JC, Fry B, Maller J, and Daly MJ. 2005. Haploview: analysis and visualization of LD and haplotype maps. Bioinformatics 21:263-265. 10.1093/bioinformatics/bth457

Braun TR, Been LF, Singhal A, Worsham J, Ralhan S, Wander GS, Chambers JC, Kooner JS, Aston CE, and Sanghera DK. 2012. A replication study of GWAS-derived lipid genes in Asian Indians: the chromosomal region 11q23.3 harbors loci contributing to triglycerides. PLoS One 7:e37056. 10.1371/journal.pone.0037056

Brooks MA, Dziembowski A, Quevillon-Cheruel S, Henriot V, Faux C, van Tilbeurgh H, and Seraphin B. 2009. Structure of the yeast Pml1 splicing factor and its integration into the RES complex. Nucleic Acids Res 37:129-143. 10.1093/nar/gkn894

Cha S, Yu H, Park AY, and Song KH. 2014. Effects of apolipoprotein A5 haplotypes on the ratio of triglyceride to high-density lipoprotein cholesterol and the risk for metabolic syndrome in Koreans. Lipids Health Dis 13:45. 10.1186/1476-511x-13-45

Dron JS, and Hegele RA. 2016. Genetics of Lipid and Lipoprotein Disorders and Traits. Curr Genet Med Rep 4:130-141. 10.1007/s40142-016-0097-y

Dron JS, and Hegele RA. 2018. Polygenic influences on dyslipidemias. Curr Opin Lipidol 29:133143. 10.1097/mol.0000000000000482

El Hajj MS, Mahfoud ZR, Al Suwaidi J, Alkhiyami D, and Alasmar AR. 2016. Role of pharmacist in cardiovascular disease-related health promotion and in hypertension and dyslipidemia 
management: a cross-sectional study in the State of Qatar. J Eval Clin Pract 22:329-340. 10.1111/jep. 12477

Ferrannini E, Balkau B, Coppack SW, Dekker JM, Mari A, Nolan J, Walker M, Natali A, and Beck-Nielsen H. 2007. Insulin resistance, insulin response, and obesity as indicators of metabolic risk. J Clin Endocrinol Metab 92:2885-2892. 10.1210/jc.2007-0334

Forte TM, Shu X, and Ryan RO. 2009. The ins (cell) and outs (plasma) of apolipoprotein A-V. $J$ Lipid Res 50 Suppl:S150-155. 10.1194/jlr.R800050-JLR200

Galcheva-Gargova Z, Konstantinov KN, Wu IH, Klier FG, Barrett T, and Davis RJ. 1996. Binding of zinc finger protein ZPR1 to the epidermal growth factor receptor. Science 272:17971802.

Goldberg IJ, Scheraldi CA, Yacoub LK, Saxena U, and Bisgaier CL. 1990. Lipoprotein ApoC-II activation of lipoprotein lipase. Modulation by apolipoprotein A-IV. J Biol Chem 265:4266-4272.

Guan F, Niu Y, Zhang T, Liu S, Ma L, Qi T, Feng J, Zuo H, Li G, Liu X, and Wang S. 2016. Twostage association study to identify the genetic susceptibility of a novel common variant of rs2075290 in ZPR1 to type 2 diabetes. Sci Rep 6:29586. 10.1038/srep29586

Hsueh WC, Nair AK, Kobes S, Chen P, Goring HHH, Pollin TI, Malhotra A, Knowler WC, Baier LJ, and Hanson RL. 2017. Identity-by-Descent Mapping Identifies Major Locus for Serum Triglycerides in Amerindians Largely Explained by an APOC3 Founder Mutation. Circ Cardiovasc Genet 10. 10.1161/circgenetics.117.001809

Lin E, Kuo PH, Liu YL, Yang AC, Kao CF, and Tsai SJ. 2016. Association and interaction of APOA5, BUD13, CETP, LIPA and health-related behavior with metabolic syndrome in a Taiwanese population. Sci Rep 6:36830. 10.1038/srep36830

Malmendier CL, Alaupovic P, and Jr HBB. 1991. Hypercholesterolemia, Hypocholesterolemia, Hypertriglyceridemia, in Vivo Kinetics. Advances in Experimental Medicine \& Biology.

Milewicz A, Tworowska U, and Demissie M. 2001. Menopausal obesity--myth or fact? Climacteric 4:273-283.

Ooi EM, Barrett PH, Chan DC, and Watts GF. 2008. Apolipoprotein C-III: understanding an emerging cardiovascular risk factor. Clin Sci (Lond) 114:611-624. 10.1042/cs20070308

Parhofer KG. 2015. Interaction between Glucose and Lipid Metabolism: More than Diabetic Dyslipidemia. Diabetes Metab J 39:353-362. 10.4093/dmj.2015.39.5.353

Pranavchand R, Kumar AS, and Reddy BM. 2017. Genetic determinants of clinical heterogeneity of the coronary artery disease in the population of Hyderabad, India. Hum Genomics 11:3. 10.1186/s40246-017-0099-1

Pranavchand R, and Reddy BM. 2017. Quantitative trait loci at the 11q23.3 chromosomal region related to dyslipidemia in the population of Andhra Pradesh, India. Lipids Health Dis 16:116. 10.1186/s12944-017-0507-5

Prevention JCfDCgo, and Adults ToDi. 2007. [Chinese guidelines on prevention and treatment of dyslipidemia in adults]. Zhonghua Xin Xue Guan Bing Za Zhi 35:390.

Rai H, Sinha N, Finn J, Agrawal S, and Mastana S. 2016. Association of serum lipids and coronary artery disease with polymorphisms in the apolipoprotein AI-CIII-AIV gene cluster. Cogent 
Med 3:1266789. 10.1080/2331205x.2016.1266789

Reddy Kilim S, and Chandala SR. 2013. A comparative study of lipid profile and oestradiol in preand post-menopausal women. $J$ Clin Diagn Res 7:1596-1598. $10.7860 / \mathrm{jcdr} / 2013 / 6162.3234$

Reichl D, and Miller NE. 1989. Pathophysiology of reverse cholesterol transport. Insights from inherited disorders of lipoprotein metabolism. Arteriosclerosis 9:785-797.

Sole X, Guino E, Valls J, Iniesta R, and Moreno V. 2006. SNPStats: a web tool for the analysis of association studies. Bioinformatics 22:1928-1929. 10.1093/bioinformatics/btl268

Stein O, Stein Y, Lefevre M, and Roheim PS. 1986. The role of apolipoprotein A-IV in reverse cholesterol transport studied with cultured cells and liposomes derived from an ether analog of phosphatidylcholine. Biochim Biophys Acta 878:7-13.

Su Y, Ma Y, Rao W, Yang G, Wang S, Fu Y, Liu Y, Zhang Y, You Y, Yu Y, and Kou C. 2016. Association Between Body Mass Index and Diabetes in Northeastern China: Based on Dose-Response Analyses Using Restricted Cubic Spline Functions. Asia Pac J Public Health 28:486-497. 10.1177/1010539516656436

Suarez-Sanchez F, Klunder-Klunder M, Valladares-Salgado A, Gomez-Zamudio J, PeraltaRomero J, Meyre D, Burguete-Garcia A, and Cruz M. 2017. APOA5 and APOA1 polymorphisms are associated with triglyceride levels in Mexican children. Pediatr Obes 12:330-336. 10.1111/ijpo.12147

Tan NC, Koh KH, Goh CC, Koh YL, and Goh SC. 2016. Asian patients with dyslipidemia in an urban population: Effect of ethnicity on their LDL-cholesterol treatment goals. $J$ Clin Lipidol 10:410-419. 10.1016/j.jacl.2015.12.014

Tokoro F, Matsuoka R, Abe S, Arai M, Noda T, Watanabe S, Horibe H, Fujimaki T, Oguri M, Kato K, Minatoguchi S, and Yamada Y. 2015. Association of a genetic variant of the ZPR1 zinc finger gene with type 2 diabetes mellitus. Biomed Rep 3:88-92. 10.3892/br.2014.379

Ueyama C, Horibe H, Yamase Y, Fujimaki T, Oguri M, Kato K, Arai M, Watanabe S, Murohara T, and Yamada Y. 2015. Association of FURIN and ZPR1 polymorphisms with metabolic syndrome. Biomed Rep 3:641-647. 10.3892/br.2015.484

Wang S, Kou C, Liu Y, Li B, Tao Y, D'Arcy C, Shi J, Wu Y, Liu J, Zhu Y, and Yu Y. 2015. Ruralurban differences in the prevalence of chronic disease in northeast China. Asia Pac J Public Health 27:394-406. 10.1177/1010539514551200

Wang Y, Liu F, Li L, Deng S, and He Z. 2017. The association between apolipoprotein A1-C3A5 gene cluster promoter polymorphisms and risk of ischemic stroke in the northern Chinese Han population. J Int Med Res 45:2042-2052. 10.1177/0300060517713517

Weiss LA, Pan L, Abney M, and Ober C. 2006. The sex-specific genetic architecture of quantitative traits in humans. Nat Genet 38:218-222. 10.1038/ng1726

Willer CJ, Schmidt EM, Sengupta S, Peloso GM, Gustafsson S, Kanoni S, Ganna A, Chen J, Buchkovich ML, Mora S, Beckmann JS, Bragg-Gresham JL, Chang HY, Demirkan A, Den Hertog HM, Do R, Donnelly LA, Ehret GB, Esko T, Feitosa MF, Ferreira T, Fischer K, Fontanillas P, Fraser RM, Freitag DF, Gurdasani D, Heikkila K, Hypponen E, Isaacs A, Jackson AU, Johansson A, Johnson T, Kaakinen M, Kettunen J, Kleber ME, Li X, Luan J, 
Lyytikainen LP, Magnusson PKE, Mangino M, Mihailov E, Montasser ME, MullerNurasyid M, Nolte IM, O'Connell JR, Palmer CD, Perola M, Petersen AK, Sanna S, Saxena R, Service SK, Shah S, Shungin D, Sidore C, Song C, Strawbridge RJ, Surakka I, Tanaka T, Teslovich TM, Thorleifsson G, Van den Herik EG, Voight BF, Volcik KA, Waite LL, Wong A, Wu Y, Zhang W, Absher D, Asiki G, Barroso I, Been LF, Bolton JL, Bonnycastle LL, Brambilla P, Burnett MS, Cesana G, Dimitriou M, Doney ASF, Doring A, Elliott P, Epstein SE, Ingi Eyjolfsson G, Gigante B, Goodarzi MO, Grallert H, Gravito ML, Groves CJ, Hallmans G, Hartikainen AL, Hayward C, Hernandez D, Hicks AA, Holm H, Hung YJ, Illig T, Jones MR, Kaleebu P, Kastelein JJP, Khaw KT, Kim E, Klopp N, Komulainen P, Kumari M, Langenberg C, Lehtimaki T, Lin SY, Lindstrom J, Loos RJF, Mach F, McArdle WL, Meisinger C, Mitchell BD, Muller G, Nagaraja R, Narisu N, Nieminen TVM, Nsubuga RN, Olafsson I, Ong KK, Palotie A, Papamarkou T, Pomilla C, Pouta A, Rader DJ, Reilly MP, Ridker PM, Rivadeneira F, Rudan I, Ruokonen A, Samani N, Scharnagl H, Seeley J, Silander K, Stancakova A, Stirrups K, Swift AJ, Tiret L, Uitterlinden AG, van Pelt LJ, Vedantam S, Wainwright N, Wijmenga C, Wild SH, Willemsen G, Wilsgaard T, Wilson JF, Young EH, Zhao JH, Adair LS, Arveiler D, Assimes TL, Bandinelli S, Bennett F, Bochud M, Boehm BO, Boomsma DI, Borecki IB, Bornstein SR, Bovet P, Burnier M, Campbell H, Chakravarti A, Chambers JC, Chen YI, Collins FS, Cooper RS, Danesh J, Dedoussis G, de Faire U, Feranil AB, Ferrieres J, Ferrucci L, Freimer NB, Gieger C, Groop LC, Gudnason V, Gyllensten U, Hamsten A, Harris TB, Hingorani A, Hirschhorn JN, Hofman A, Hovingh GK, Hsiung CA, Humphries SE, Hunt SC, Hveem K, Iribarren C, Jarvelin MR, Jula A, Kahonen M, Kaprio J, Kesaniemi A, Kivimaki M, Kooner JS, Koudstaal PJ, Krauss RM, Kuh D, Kuusisto J, Kyvik KO, Laakso M, Lakka TA, Lind L, Lindgren CM, Martin NG, Marz W, McCarthy MI, McKenzie CA, Meneton P, Metspalu A, Moilanen L, Morris AD, Munroe PB, Njolstad I, Pedersen NL, Power C, Pramstaller PP, Price JF, Psaty BM, Quertermous T, Rauramaa R, Saleheen D, Salomaa V, Sanghera DK, Saramies J, Schwarz PEH, Sheu WH, Shuldiner AR, Siegbahn A, Spector TD, Stefansson K, Strachan DP, Tayo BO, Tremoli E, Tuomilehto J, Uusitupa M, van Duijn CM, Vollenweider P, Wallentin L, Wareham NJ, Whitfield JB, Wolffenbuttel BHR, Ordovas JM, Boerwinkle E, Palmer CNA, Thorsteinsdottir U, Chasman DI, Rotter JI, Franks PW, Ripatti S, Cupples LA, Sandhu MS, Rich SS, Boehnke M, Deloukas P, Kathiresan S, Mohlke KL, Ingelsson E, and Abecasis GR. 2013. Discovery and refinement of loci associated with lipid levels. Nat Genet 45:1274-1283. 10.1038/ng.2797

Wu FC, and von Eckardstein A. 2003. Androgens and coronary artery disease. Endocr Rev 24:183217. 10.1210/er.2001-0025

Wu Y, Yu S, Wang S, Shi J, Xu Z, Zhang Q, Fu Y, Qi Y, Liu J, Fu R, Kou C, and Yaqin Y. 2015. Zinc Finger Protein 259 (ZNF259) Polymorphisms are Associated with the Risk of Metabolic Syndrome in a Han Chinese Population. Clin Lab 61:615-621.

Wu Y, Yu Y, Zhao T, Wang S, Fu Y, Qi Y, Yang G, Yao W, Su Y, Ma Y, Shi J, Jiang J, and Kou C. 2016. Interactions of Environmental Factors and APOA1-APOC3-APOA4-APOA5 
432

433

434

435

436

437

438

439

440

441

442

443

444

445

446

447

448

449

450

451

452

453

454

455

456

457

458

459

460

461

462

463

Gene Cluster Gene Polymorphisms with Metabolic Syndrome. PLoS One 11:e147946. 10.1371/journal.pone. 0147946

Xu X, Li Y, Huang Y, Ye H, Han L, Ji H, Chen X, Wu N, Jiang D, Xu L, Dai D, Duan S, and Chen X. 2018. Impact of gender and age on the association of the BUD13-ZNF259 rs964184 polymorphism with coronary heart disease. Anatol $J$ Cardiol 19:42-49. 10.14744/AnatolJCardiol.2017.8002

Yamada Y, Sakuma J, Takeuchi I, Yasukochi Y, Kato K, Oguri M, Fujimaki T, Horibe H, Muramatsu M, Sawabe M, Fujiwara Y, Taniguchi Y, Obuchi S, Kawai H, Shinkai S, Mori S, Arai T, and Tanaka M. 2017. Identification of eight genetic variants as novel determinants of dyslipidemia in Japanese by exome-wide association studies. Oncotarget 8:38950-38961. 10.18632/oncotarget.17159

Y in RX, Li YY, and Lai CQ. 2011. Apolipoprotein A1/C3/A5 haplotypes and serum lipid levels. Lipids Health Dis 10:140. 10.1186/1476-511x-10-140

You Y, Wu YH, Zhang Y, Zhang L, Song Y, Bai W, Li Y, Yu Y, and Kou C. 2018. Effects of polymorphisms in APOA5 on the plasma levels of triglycerides and risk of coronary heart disease in Jilin, northeast China: a case-control study. BMJ Open 8:e020016. 10.1136/bmjopen-2017-020016

You Y, Yu Y, Wu Y, Rao W, Zhang Y, Liu Y, Yang G, Fu Y, Shi J, and Kou C. 2017. Association Study between Ghrelin Gene Polymorphism and Metabolic Syndrome in a Han Chinese Population. Clin Lab 63:175-181. 10.7754/Clin.Lab.2016.160715

Zhang M, Deng Q, Wang L, Huang Z, Zhou M, Li Y, Zhao Z, Zhang Y, and Wang L. 2018. Prevalence of dyslipidemia and achievement of low-density lipoprotein cholesterol targets in Chinese adults: A nationally representative survey of 163,641 adults. Int $J$ Cardiol 260:196-203. 10.1016/j.ijcard.2017.12.069

Zhou L, He M, Mo Z, Wu C, Yang H, Yu D, Yang X, Zhang X, Wang Y, Sun J, Gao Y, Tan A, He Y, Zhang H, Qin X, Zhu J, Li H, Lin X, Zhu J, Min X, Lang M, Li D, Zhai K, Chang J, Tan W, Yuan J, Chen W, Wang Y, Wei S, Miao X, Wang F, Fang W, Liang Y, Deng Q, Dai X, Lin D, Huang S, Guo H, Lilly Zheng S, Xu J, Lin D, Hu FB, and Wu T. 2013. A genome wide association study identifies common variants associated with lipid levels in the Chinese population. PLoS One 8:e82420. 10.1371/journal.pone.0082420 


\section{Table 1 (on next page)}

Basic characteristics and distribution of dyslipidemia of included subjects 
Table 1 Basic characteristics and distribution of dyslipidemia of included subjects

\begin{tabular}{ccccc}
\hline Characteristics & $\begin{array}{c}\text { Total } \\
(\mathrm{n}=3850)\end{array}$ & $\begin{array}{c}\text { Male } \\
(\mathrm{n}=1927)\end{array}$ & $\begin{array}{c}\text { Female } \\
(\mathrm{n}=1923)\end{array}$ & $P$ \\
\hline Age (year) & $49.54(9.58)$ & $49.93(9.75)$ & $49.16(9.39)$ & $\mathbf{0 . 0 1 3}$ \\
Waist (cm) & $82.73(11.22)$ & $84.74(11.25)$ & $80.72(10.83)$ & $<\mathbf{0 . 0 0 1}$ \\
BMI (kg/m $\left.{ }^{2}\right)$ & $24.40(3.96)$ & $24.38(3.93)$ & $24.42(4.00)$ & 0.734 \\
Hypertriglyceridemia, n (\%) & $1110(28.8)$ & $664(34.5)$ & $446(23.2)$ & $<\mathbf{0 . 0 0 1}$ \\
Hypercholesterolemia, n (\%) & $444(11.5)$ & $214(11.1)$ & $230(12.0)$ & 0.406 \\
High LDL, n (\%) & $352(9.1)$ & $145(7.5)$ & $207(10.8)$ & $<\mathbf{0 . 0 0 1}$ \\
Reduced HDL, n (\%) & $719(18.7)$ & $450(23.4)$ & $269(14.0)$ & $<\mathbf{0 . 0 0 1}$ \\
Dyslipidemia, $\mathrm{n}(\%)$ & $1586(41.2)$ & $880(45.7)$ & $706(36.7)$ & $<\mathbf{0 . 0 0 1}$ \\
\hline
\end{tabular}

2 Note:

3 BMI, body mass index

4 Means (standard deviation) for age, waist and BMI, a $P$ value in bold indicated differences were 5 significant between males and females $(P<0.05)$. 


\section{Table 2 (on next page)}

Association between genotypes of the six SNPs and dyslipidemia stratified by genders 
Table 2 Association between genotypes of the six SNPs and dyslipidemia stratified by genders

\begin{tabular}{|c|c|c|c|c|}
\hline SNPs & $\begin{array}{c}\text { Hypertriglyceridemia } \\
(\mathrm{n}=1110) \\
\text { OR }(95 \% \mathrm{CI})^{\mathrm{a}} \\
\end{array}$ & $\begin{array}{c}\text { Hypercholesterolemia } \\
(\mathrm{n}=444) \\
\text { OR }(95 \% \mathrm{CI}) \\
\end{array}$ & $\begin{array}{c}\text { High LDL } \\
(\mathrm{n}=352) \\
\text { OR }(95 \% \mathrm{CI}) \\
\end{array}$ & $\begin{array}{c}\text { Reduced HDL } \\
(\mathrm{n}=719) \\
\text { OR }(95 \% \mathrm{CI}) \\
\end{array}$ \\
\hline \multicolumn{5}{|l|}{ Overall } \\
\hline \multicolumn{5}{|l|}{ APOA1-rs5072 } \\
\hline TC+TT vs CC & $1.36(1.15-1.60)$ & $1.02(0.83-1.25)$ & $0.84(0.67-1.05)$ & $1.16(0.97-1.39)$ \\
\hline \multicolumn{5}{|l|}{$A P O A 4-\mathrm{rs} 5104$} \\
\hline $\mathrm{GA}+\mathrm{GG}$ vs $\mathrm{AA}$ & $1.25(1.06-1.48)$ & $1.01(0.82-1.25)$ & $0.82(0.65-1.03)$ & $1.24(1.03-1.49)$ \\
\hline \multicolumn{5}{|l|}{$A P O A 3-\mathrm{rs} 5128$} \\
\hline $\mathrm{GC}+\mathrm{CC}$ vs GG & $1.33(1.13-1.57)$ & $1.02(0.83-1.25)$ & $0.84(0.67-1.05)$ & $1.16(0.97-1.39)$ \\
\hline \multicolumn{5}{|l|}{$A P O A 5$-rs651821 } \\
\hline $\mathrm{CT}+\mathrm{CC}$ vs TT & $2.01(1.71-2.38)$ & $1.23(1.00-1.50)$ & $1.00(0.80-1.25)$ & $1.90(1.59-2.28)$ \\
\hline \multicolumn{5}{|l|}{ ZPR1-rs2075294 } \\
\hline GT+TT vs GG & $0.89(0.74-1.07)$ & $0.83(0.66-1.05)$ & $0.87(0.67-1.13)$ & $0.91(0.75-1.12)$ \\
\hline \multicolumn{5}{|l|}{$B U D 13-$} \\
\hline $\mathrm{CT}+\mathrm{TT}$ vs CC & $0.75(0.59-0.95)$ & $1.02(0.77-1.35$ & $0.99(0.73-1.36)$ & $0.70(0.54-0.92)$ \\
\hline \multicolumn{5}{|l|}{ Males } \\
\hline \multicolumn{5}{|l|}{ APOA1-rs5072 } \\
\hline TC+TT vs CC & $1.34(1.06-1.69)$ & $1.16(0.86-1.56)$ & $0.81(0.58-1.15)$ & $1.19(0.94-1.52)$ \\
\hline \multicolumn{5}{|l|}{ APOA4-rs5104 } \\
\hline $\mathrm{GA}+\mathrm{GG}$ vs $\mathrm{AA}$ & $1.28(1.01-1.63)$ & $1.14(0.84-1.54)$ & $0.77(0.54-1.09)$ & $1.28(1.00-1.63)$ \\
\hline \multicolumn{5}{|l|}{$A P O A 3-$ rs 5128} \\
\hline $\mathrm{GC}+\mathrm{CC}$ vs $\mathrm{GG}$ & $1.31(1.04-1.65)$ & $1.20(0.89-1.62)$ & $0.85(0.60-1.20)$ & $1.16(0.91-1.47)$ \\
\hline \multicolumn{5}{|l|}{ APOA5-rs651821 } \\
\hline $\mathrm{CT}+\mathrm{CC}$ vs TT & $1.78(1.41-2.26)$ & $1.16(0.86-1.56)$ & $0.77(0.55-1.10)$ & $1.61(1.27-2.05)$ \\
\hline \multicolumn{5}{|l|}{ ZPR1-rs2075294 } \\
\hline GT+TT vs GG & $0.99(0.76-1.30)$ & $0.68(0.48-0.98)$ & $0.61(0.39-0.95)$ & $1.03(0.78-1.35)$ \\
\hline \multicolumn{5}{|l|}{$B U D 13-$} \\
\hline $\mathrm{CT}+\mathrm{TT}$ vs CC & $0.87(0.62-1.20)$ & $1.24(0.84-1.83)$ & $1.68(1.10-2.57)$ & $0.74(0.52-1.05)$ \\
\hline \multicolumn{5}{|l|}{ Females } \\
\hline APOA1-rs5072 & & & & \\
\hline
\end{tabular}




\begin{tabular}{|c|c|c|c|c|}
\hline TC+TT vs CC & $1.39(1.09-1.76)$ & $0.92(0.69-1.22)$ & $0.87(0.65-1.17)$ & $1.10(0.84-1.45)$ \\
\hline GA + GG vs AA & $1.22(0.96-1.55)$ & $0.91(0.68-1.22)$ & $0.86(0.64-1.17)$ & $1.18(0.89-1.55)$ \\
\hline APOA3-rs5128 & & & & \\
\hline $\begin{array}{l}\mathrm{GC}+\mathrm{CC} \text { vs } \mathrm{GG} \\
A P O A 5 \text {-rs651821 }\end{array}$ & $1.37(1.08-1.74)$ & $0.89(0.67-1.18)$ & $0.85(0.63-1.15)$ & $1.14(0.87-1.50)$ \\
\hline $\begin{array}{l}\text { CT+CC vs TT } \\
\text { ZPR1-rs2075294 }\end{array}$ & $2.26(1.77-2.88)$ & $1.27(0.95-1.69)$ & $1.19(0.89-1.60)$ & $2.29(1.73-3.04)$ \\
\hline $\begin{array}{l}\text { GT+TT vs GG } \\
B U D 13-\end{array}$ & $0.82(0.63-1.07)$ & $1.01(0.73-1.38)$ & $1.11(0.80-1.53)$ & $0.78(0.57-1.07)$ \\
\hline $\mathrm{CT}+\mathrm{TT}$ vs $\mathrm{CC}$ & $0.66(0.46-0.94)$ & $0.94(0.62-1.42)$ & $0.64(0.39-1.03)$ & $0.67(0.44-1.02)$ \\
\hline
\end{tabular}

2 Note:

3 SNP, single nucleotide polymorphism

4 BMI, body mass index

OR, odds ratio

CI, confidence interval

ORs $(95 \% \mathrm{CI})$ were shown in bold if corresponding $P$ values were no more than $0.008(0.05 / 6)$.

8 The genetic model in this association analysis was the Dominant Model.

9 (a) ORs and 95\% CI were adjusted for sex, age, waist circumference and BMI in the overall group; ORs and 95\% CI were adjusted for 10 age, waist circumference and BMI in the male and female groups. 


\section{Table 3(on next page)}

Association between APOA1/C3/A4/A5-ZPR1-BUD13 gene cluster haplotypes and the risk for dyslipidemia 
1 Table 3 Association between $A P O A 1 / C 3 / A 4 / A 5-Z P R 1-B U D 13$ gene cluster haplotypes and the 2 risk for dyslipidemia

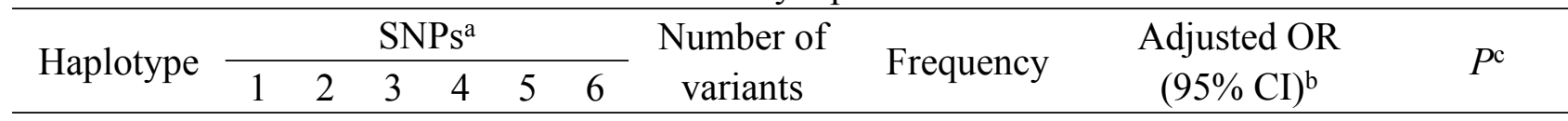

\section{Overall}

$\begin{array}{ccccccc}1 & \text { C } & \text { A } & \text { G } & \text { T } & \text { G } & \text { C } \\ 2 & \text { T } & \text { G } & \text { C } & \text { C } & \text { G } & \text { C } \\ 3 & \text { C } & \text { A } & \text { G } & \text { C } & \text { G } & \text { C } \\ 4 & \text { C } & \text { A } & \text { G } & \text { T } & \text { T } & \text { C } \\ 5 & \text { C } & \text { A } & \text { G } & \text { T } & \text { G } & \text { T } \\ 6 & \text { T } & \text { G } & \text { C } & \text { T } & \text { T } & \text { C } \\ 7 & \text { T } & \text { G } & \text { C } & \text { T } & \text { G } & \text { C } \\ 8 & \text { T } & \text { A } & \text { C } & \text { T } & \text { G } & \text { C } \\ 9 & \text { C } & \text { G } & \text { G } & \text { C } & \text { G } & \text { C } \\ 10 & \text { C } & \text { G } & \text { G } & \text { T } & \text { G } & \text { C } \\ 11 & \text { T } & \text { A } & \text { C } & \text { C } & \text { G } & \text { C } \\ 12 & \text { C } & \text { G } & \text { G } & \text { T } & \text { T } & \text { C }\end{array}$

Rare $^{\mathrm{d}}$

Males

$\begin{array}{ccccccc}1 & \text { C } & \text { A } & \text { G } & \text { T } & \text { G } & \text { C } \\ 2 & \text { T } & \text { G } & \text { C } & \text { C } & \text { G } & \text { C } \\ 3 & \text { C } & \text { A } & \text { G } & \text { C } & \text { G } & \text { C } \\ 4 & \text { C } & \text { A } & \text { G } & \text { T } & \text { G } & \text { T } \\ 5 & \text { C } & \text { A } & \text { G } & \text { T } & \text { T } & \text { C } \\ 6 & \text { T } & \text { G } & \text { C } & \text { T } & \text { G } & \text { C } \\ 7 & \text { T } & \text { G } & \text { C } & \text { T } & \text { T } & \text { C } \\ 8 & \text { T } & \text { A } & \text { C } & \text { T } & \text { G } & \text { C } \\ 9 & \text { C } & \text { G } & \text { G } & \text { C } & \text { G } & \text { C } \\ 10 & \text { C } & \text { G } & \text { G } & \text { T } & \text { G } & \text { C } \\ 11 & \text { C } & \text { G } & \text { G } & \text { T } & \text { T } & \text { C }\end{array}$

Rare $^{\mathrm{d}}$

Females

$\begin{array}{ccccccccccc}1 & \text { C } & \text { A } & \text { G } & \text { T } & \text { G } & \text { C } & 0 & 0.3932 & 1.00 & - \\ 2 & \text { T } & \text { G } & \text { C } & \text { C } & \text { G } & \text { C } & 4 & 0.1199 & 1.89(1.45-2.45) & <\mathbf{0 . 0 0 0 1} \\ 3 & \text { C } & \text { A } & \text { G } & \text { C } & \text { G } & \text { C } & 1 & 0.1084 & 1.71(1.29-2.28) & \mathbf{2 e - 0 4} \\ 4 & \text { C } & \text { A } & \text { G } & \text { T } & \text { T } & \text { C } & 1 & 0.0720 & 1.12(0.80-1.59) & 0.50 \\ 5 & \text { T } & \text { G } & \text { C } & \text { T } & \text { T } & \text { C } & 3 & 0.0638 & 1.04(0.72-1.50) & 0.85 \\ 6 & \text { T } & \text { G } & \text { C } & \text { T } & \text { G } & \text { C } & 3 & 0.0620 & 0.68(0.44-1.06) & 0.09 \\ 7 & \text { C } & \text { A } & \text { G } & \text { T } & \text { G } & \text { T } & 1 & 0.0602 & 0.80(0.55-1.15) & 0.23 \\ 8 & \text { T } & \text { A } & \text { C } & \text { T } & \text { G } & \text { C } & 2 & 0.0237 & 0.67(0.33-1.35) & 0.26 \\ 9 & \text { C } & \text { G } & \text { G } & \text { C } & \text { G } & \text { C } & 2 & 0.0216 & 1.94(1.05-3.58) & 0.033\end{array}$




\begin{tabular}{ccccccccccc}
10 & $\mathrm{C}$ & $\mathrm{G}$ & $\mathrm{G}$ & $\mathrm{T}$ & $\mathrm{G}$ & $\mathrm{C}$ & 1 & 0.0149 & $1.39(0.58-3.32)$ & 0.45 \\
11 & $\mathrm{~T}$ & $\mathrm{~A}$ & $\mathrm{C}$ & $\mathrm{C}$ & $\mathrm{G}$ & $\mathrm{C}$ & 3 & 0.0144 & $2.76(1.30-5.85)$ & 0.0081 \\
12 & $\mathrm{C}$ & $\mathrm{G}$ & $\mathrm{G}$ & $\mathrm{T}$ & $\mathrm{T}$ & $\mathrm{C}$ & 2 & 0.0129 & $1.48(0.69-3.16)$ & 0.31 \\
13 & $\mathrm{~T}$ & $\mathrm{G}$ & $\mathrm{C}$ & $\mathrm{T}$ & $\mathrm{G}$ & $\mathrm{T}$ & 4 & 0.0115 & $1.52(0.61-3.75)$ & 0.37 \\
Rare $^{\mathrm{d}}$ & - & - & - & - & - & - & & 0.0214 & $1.16(0.58-2.30)$ & 0.67 \\
\hline
\end{tabular}

3 Note:

4 SNP, single nucleotide polymorphism

5 BMI, body mass index

6 OR, odds ratio

7 CI, confidence interval

8 (a) SNPs are as follows: 1, rs5072; 2, rs5104; 3, rs5128; 4, rs651821; 5, rs2075294; 6, rs10488698.

9 (b) ORs were adjusted for sex, age, BMI and waist circumference in the overall group and were 10 adjusted for age, BMI and waist circumference in males and females.

11 (c) $P$ values of less than 0.008 were considered to be significant after Bonferroni correction and 12 were presented in bold.

13 (d) Rare: haplotypes with frequencies $<0.01$. 
Figure 1

Linkage disequilibrium analysis of six SNPs

The value of $D^{\prime}$ chosen as cut-off for linkage disequilibrium was 0.75 .
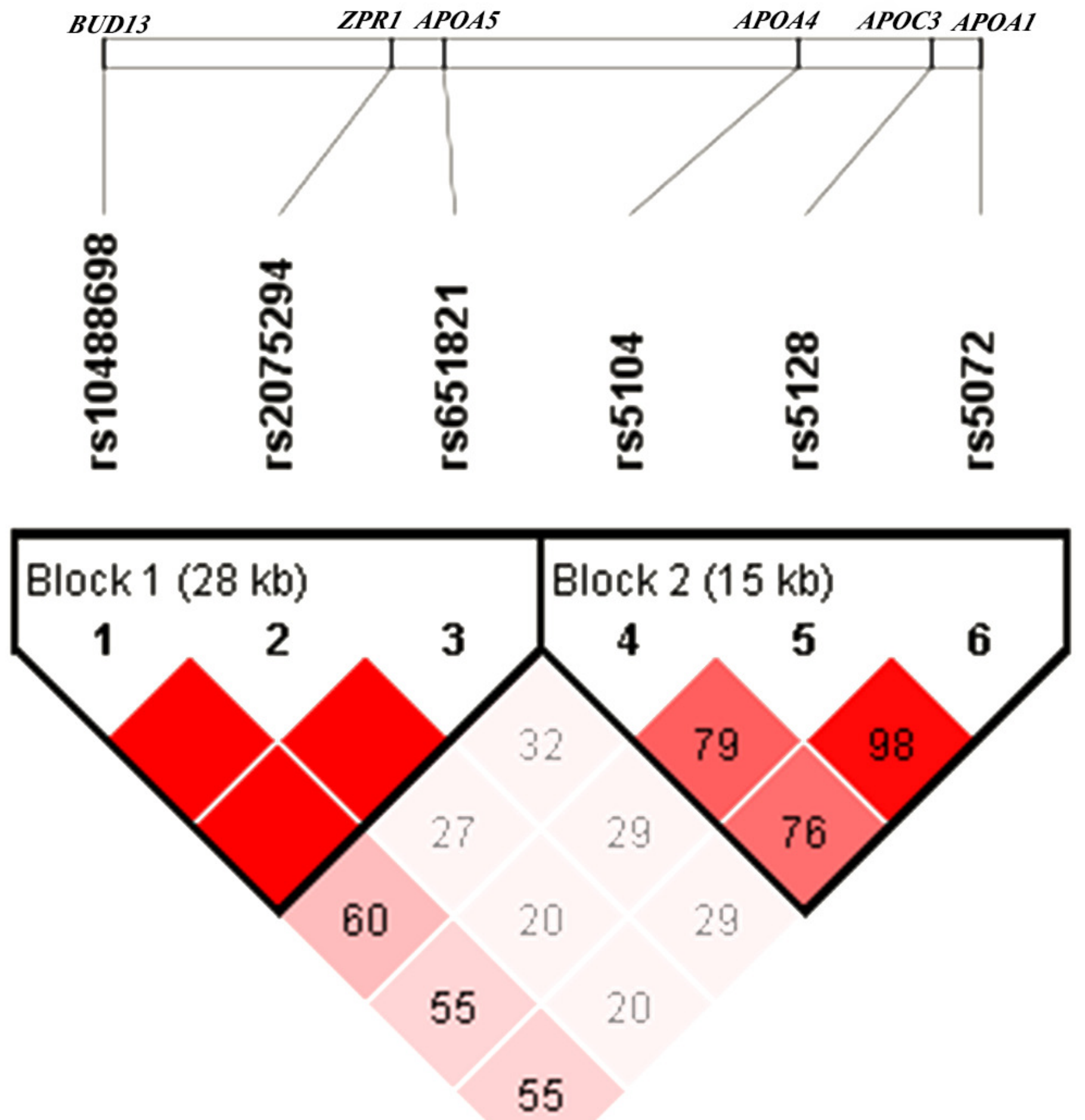